\title{
Isolated Para-Aortic Lymph Nodes Recurrence in Carcinoma Cervix
}

\author{
Ghimire $\mathrm{S}^{1}$, Hamid S, ${ }^{2}$ Rashid $\mathrm{A}^{2}$ \\ ${ }^{1}$ Bhaktapur Cancer Hospital, Bhaktapur, Nepal, ${ }^{2}$ Shaukat Khanum Memorial Cancer Hospital, Lahore, Pakistan.
}

\begin{abstract}
Background: To evaluate the presentation of isolated para-aortic lymph node recurrence after definitive treatment, and to analyze the effect of salvage treatment on the survival rate.

Methods: A retrospective study was done for patients with invasive carcinoma of the uterine cervix treated with various modalities of treatment at Shaukat Khanum Memorial Cancer Hospital Research Center Lahore Pakistan from December, 1999 to August 2004.

Results: The incidence of radiographically detected para aortic lymph node recurrence was $3.7 \%$ by FIGO stage at original diagnosis was $3(50 \%)$ in stage IIIB, $2(33.3 \%)$ in post hysterectomy status and $1(16.7 \%)$ in stage IB cervical carcinoma. Total 4 (66.7\%) patients with para-aortic lymph nodes recurrences were identified with in the first 12 months after the initial treatment and 2(33.3\%) had their recurrence after 12 month. Leg swelling, low back pain and hydronephrosis were observed when inquired for the presentation in those patients with recurrence in paraarotic lymph nodes. Hydronephrosis was the commonest finding (50\%) either alone or in association with other symptoms. And $3(50 \%)$ of the patient were died within two year of the diagnosis of recurrence. Three patients are still on follow up, one with progressive disease in liver and one with chronic renal failure, and one asymptomatic. Patient who received radiation alone had overall survival of 3.16 months after the recurrence compared with $>12$ months overall survival for those treated with chemotherapy.

Conclusions: Symptoms like leg swelling, low back pain and hydronephrosis helps a lot to detect the early paraaortic recurrence, hydronephrosis being commonest. Although the number of patients with para-aortic recurrence were very small and the follow up time was too short to make any recommendations for salvage treatment or survival advantages, as a definitive treatment for isolated para aortic lymph nodes we can offer both radiotherapy or chemotherapy.

Key words: cervix, carcinoma, lymph nodes, para-aortic, recurrence.
\end{abstract}

\section{INTRODUCTION}

Carcinoma of the uterine cervix has the highest incidence of gynecological cancers in Asia and is one of the most common cancers of the female genital tract. ${ }^{1,2}$ Worldwide, nearly one-half million new diagnoses of, and an associated one-quarter million deaths from, invasive cervical cancer have been estimated to occur. ${ }^{3,4}$ In the United States, 12,900 new diagnoses of, and an associated 4400 deaths from, cervical carcinoma have been estimated to occur annually. ${ }^{5}$ Early detection and improved radiation and surgical techniques used with 
in the past four decades in the management of this malignancy have resulted in better control of the pelvic tumor and longer patient survival. These improved results have been accompanied by a higher incidence of distant metastases. ${ }^{6}$ Definitive irradiation plays an important role in the treatment of these patients, especially those of an advanced stage. The efficacy of treatment is related to clinical stage, tumor size, and the dose of irradiation. ${ }^{9}$ The prognosis for cervical carcinoma after irradiation is inversely related to its stage. ${ }^{10}$ For all stages, radiation therapy (RT) provides 5 -year survival and disease free survival rates of $61 \%$ and $73 \%$, respectively. ${ }^{12}$ It is estimated from published results that around $30 \%$ of patients show recurrence at local or distant sites, or both, after primary RT. ${ }^{12,13} \mathrm{~A}$ distinguishing characteristic of tumor metastasis in cervical carcinoma is its predictable and orderly spread along the regional lymphatic chains. The rate of para-aortic lymph node (PALN) metastasis increases with clinical stage and was reported as $5 \%, 16 \%$, and $25 \%$ in one surgical study for Stage I, II, and III disease, respectively. ${ }^{1}$ The prognosis of biopsy-proven PALN-positive patients at the time of initial diagnosis is poorer than that of PALN-negative patients in cervical carcinoma, and the presence or absence of PALN metastasis is a more important prognostic factor than clinical stage. ${ }^{7}$ Moreover, the prognosis of exclusive PALN recurrence after definitive radiation therapy is very poor. Grigsby et al. reported that such patients died within 2 years of recurrence, despite salvage radiation therapy, and PALN recurrence was regarded as a uniformly fatal condition. ${ }^{8}$ Salvage therapies of various modalities are of limited effectiveness for both local and distant recurrences. ${ }^{10}$ Distant metastasis comprises about half of the recurrent cervical carcinoma, and has a lower survival rate than local recurrence. ${ }^{11}$ Lymph node metastasis in the initial diagnosis is associated with a significantly poorer survival rate for patients undergoing primary irradiation or surgery. ${ }^{11,13-15}$ Para aortic lymph node (PALN) recurrence is frequently associated with other distant metastasis and is a fatal disease. ${ }^{16}$

\section{METHODS}

A retrospective study was done for patients with invasive carcinoma of the uterine cervix treated with various modalities of treatment at Shaukat Khanum Memorial Cancer Hospital Research Center Lahore Pakistan from December, 1999 to August 2004. Hopital permission was taken for the study. The records with presenting with histological proven carcinoma of the uterine cervix, stage ( A to IVB, according to the International Federation of Gynecology and Obstetrics (FIGO) classification were being treated with concurrent chemo-radiation or radiation only at Shaukat Khanum Memorial Cancer Hospital and Research Center. Out of the 161 patient,
6 of which had radiographically detected isolated para aortic lymph node (PALN) metastases were identified. Patients with histological proven i.e surgical specimen or image suspicious para- aortic lymph node metastases before initiation of or during primary treatment with RT were not included in this study. Patients were initially evaluated with physical and pelvic examinations, chemistry profile, routine blood work, chest $\mathrm{x}$ - ray, CT/ MRI scans.

Patients were treated with a combination of external irradiation with or without systemic chemotherapy and intracavitory brachytherapy. The treatment method was to give 40-50 Gy whole pelvic irradiations with $\mathrm{CO} 60$ or 6-MV X-ray by parallel-opposed anteroposterior or fourfield box technique. The para-aortic lymph nodes were not routinely included in the treatment field. Mid line block was added at $40 \mathrm{~Gy}$. Parametria were boosted up to $58 \mathrm{~Gy}$ by a parallel-opposed anteroposterior field in patients with Stage IIB or higher stages. The daily fraction was 1.8-2 Gy, 5 fractions per week. In tumors with lower vaginal extension, bladder or rectal invasion, or a persistent bulky tumor after $44 \mathrm{~Gy}$ of irradiation, external beam doses to the low pelvis were increased to 50-54 Gy without central block and followed by brachytherapy. If chemotherapy was given, it was used concurrently with XRT on weekly basis.

During therapy patients were evaluated for toxicity and response weekly and at therapy completion. Patients were follow up in the outpatient clinic with pelvic examination 6 weekly for first 3 months, then at an interval of 3 months for 3 years, and then every 6 months thereafter. Pelvic-abdominal computed tomography (CT) or magnetic resonance imaging (MRI) scan and chest X-ray were done routinely 6 months after completing treatment and then annually or whenever recurrence was suspected.

An isolated PALN recurrence: An unequivocally enlarged PALN by image study, with neither a concomitant loco regional relapse nor another distant recurrent lesion being found at the same time. A total of 6 patients were diagnosed having isolated PALN recurrence and enrolled in this study. Two modalities of treatment were used, three patient received local XRT only to para aortics \& three patients received chemotherapy alone.

The salvage irradiation to the PALN recurrence was done with $\mathrm{Co}^{60}$. A $1-\mathrm{cm}$ gap from the previously irradiated upper margin was included to avoid an overlap with and an excessive dose to the small intestine. A total dose of $30 \mathrm{~Gy}$ was given with the fraction size of 300 cGy per day with parallel opposed anteroposterior / poster anterior field, 5 days a week, to 10 fractions. 
Cisplatin with taxol, carboplatin with taxol at three weeks interval were given for 2 and 3 cycles according to the response of the patient.

The time to recurrence was defined as the length of time from the initial diagnosis to the time of radiological evidence of PALN recurrence. Survival after recurrence was defined as the length of time from the recurrence to the patient's death or last follow up. Survival curves were calculated according to the Kaplan-Meier method and survival analysis was performed using the log-rank test.

\section{RESULTS}

Out of $6(3.7 \%)$ cervical cancer patients were found to have isolated PALN recurrence following radical treatment with radiotherapy and chemotherapy were enrolled for further analysis. All six patients had squamous cell carcinoma, and the mean age at the time of recurrence was 54 years (Range: 35-82 years).

Four cases of recurrence $(66.66 \%)$ were observed within 12 months after the initial diagnosis and treatment of disease. By 21 months, another recurrence was identified, representing $33.33 \%$ of cases. The Median time between the initial treatment and recurrence to PALN was 21 months.

Low backache, leg edema and hydronephrosis were three clinical findings among which all or either of them were present in all 6 patient diagnosed as having isolated para-aortic recurrence.

$50 \%$ ( 3) of patients with recurrent cervical carcinoma in the para aortic lymph nodes were isolated with hydronephrosis. One of them developed all three symptoms and 1 had leg swelling and hydronephrosis detected by CT scan. Two of them had backache associated with hydronephrosis and one patient had no symptoms.

All the six patients were diagnosed radiographically by comparing the CT scans of abdomen \& pelvis with the CT scan abdomen and pelvis at initial presentation.

Among the six patients, 3 died of cervical cancer recurrence within two year of the diagnosis.

Out of three, $33.33 \%$ of patients $(1 / 3)$ died in less than 10 months of diagnosis and two died within two years of treatment of recurrence. Median survival was 21 months

As salvage therapy patient recieving XRT and combination of various chemotherapies, out of three alive patients on follow up, one with progressive disease in liver and one with chronic renal failure, and one is asymptomatic. Two are alive post treatment of recurrence without disease and one patient alive with the disease till study period. The patient who received two drugs chemotherapy only, she survived for about nine months post treatment of recurrence.

Patient who developed recurrence beyond 12 months of initial diagnosis of recurrence had median survival of 21 months, compared with 8 months for those whose recurrence was documented within 12 months of original diagnosis $(\mathrm{P}=0.01)$.

\section{DISCUSSION}

The incidence, after definitive therapy for invasive carcinoma of the uterine cervix, of radiographically detected isolated disease recurrence in the PALNs varies from $2 \%$ to $12 \%$. Carl et al. ${ }^{17}$ found para-aortic recurrences in $20(12 \%)$ of 173 patients. In two larger series, with a combined total of 2087 patients, the incidence of isolated para-aortic recurrence was approximately $2 \%$. ${ }^{2,8}$ The incidence in Anurag $\mathrm{K}$ Singh study was $1.7 \%$. While we found it in $3.7 \%$ of patients.

Definitive irradiation is an effective treatment for cervical carcinoma at any stage. All patients, after irradiation, whatever the clinical stage of their primary cervical carcinoma, shows tumor recurrence in about one-third of patients at local and/or distant sites. Most of these recurrences are refractory to salvage treatments. PALN recurrence is one of the distant recurrences, which is often associated with other simultaneous metastatic lesions and usually shows a poor outcome. ${ }^{18}$ Isolated PALN recurrence after definitive irradiation is still thought to be associated with a poor outcome. ${ }^{8}$

Chemotherapy plays an adjuvant role in the treatment of recurrent and primary cervical carcinoma. ${ }^{13,14,19}$ Concurrent chemo radiation using a cisplatin-based regimen is shown in the literature to have an acceptable toxicity and shows a high response rate in both primary and recurrent cervical carcinoma. ${ }^{16}$

The main problem in PALN recurrence is the continued development of distant metastases, despite salvage treatment for PALN recurrence. Regular follow-up after PALN treatment is necessary to detect subsequent metastasis by investigations and clinical examination. ${ }^{2,8}$

The clinical triad of recurrent cervix carcinoma includes sciatica, leg edema, and hydronephrosis. These are important symptoms and signs that were once used to predict PALN recurrence. ${ }^{8}$ These are the results from mass effect of the para-aortic node recurrence causing compression of the nerve roots and compression and obstruction of the ureter and lymphatics. Paunier et al, ${ }^{20}$ identified a "triad" of clinical findings that occur in active regional pelvic disease: leg edema, 
hydronephrosis and sciatic pain. Seventy-five percent of Grigsby series patients with para-aortic recurrence presented with sciatic pain, $45 \%$ presented with leg edema, and $65 \%$ presented with hydronephrosis. All three symptoms were documented in $40 \%$ of cases. In our study, we found all three symptoms in $16.6 \%$ of patients, hydronephrosis in $50 \%$ of the patient, only back pain with or without hydronephrosis in $33.33 \%$ and no symptoms in $16.6 \%$ of patients. When even one of these clinical pictures is identified in a patient with a history of cervical carcinoma, aggressive screening with frequent CT scans, magnetic resonance (MR) scans, or lymph angiograms should be performed regardless of the initial stage of the carcinoma.

\section{CONCLUSIONS}

For the early detection of the recurrence we should kept in mind of the common symptoms like leg swelling, low back pain and hydronephrosis which are found to be the main pointers towards the disease and helps a lot to detect the early para-aortic recurrence, hydronephrosis being commonest.

Patient with longer remission time after primary treatment has more survival benefits.

Although the number of patients with para-aortic recurrence were very small and the follow up time was too short to make any recommendations for salvage treatment or survival advantages, as a definitive treatment for isolated para aortic lymph nodes we can offer both radiotherapy or chemotherapy.

A small percentage of patients will fail in the paraaortic lymph nodes only; however, even with treatment, this condition is uniformly fatal. Symptoms like leg swelling, low back pain and the radiological follow up helps the lot to detect the early para-aortic recurrence. Various modalities of treatment can be opted after the recurrence: including local XRT and systemic chemotherapy. Although the numbers of patients with para-aortic recurrence were very small and the follow up time was too short to make any recommendations for salvage treatment or survival advantages after salvage treatment.

\section{REFERENCES}

1. Kim JS, Kim SY, Kim KH, et al. Hyper fractionated radiotherapy with concurrent chemotherapy for para-aortic lymph node recurrence in carcinoma of the cervix. International Journal of Radiation Oncology*Biology* Physics. 2003 Apr;55(5):1247-53.

2. Chou $\mathrm{HH}$, Wang $\mathrm{CC}$, Lai $\mathrm{CH}$, et al. Isolated para-aortic lymph node recurrence after definitive irradiation for cervical carcinoma. International Journal of Radiation Oncology*Biology* Physics. 2001 Oct;51(2):442- 8.
3. Parkin DM, Bray F, Ferlay J, et al. estimating the world cancer burden: Globocan 2000. Int J Cancer. 2001;94:153-156.

4. National Institutes of Health consensus development conference statement on cervical cancer. Gynecol Oncol. 1997;66(3)35161 .

5. Greenlee RT, Hill-Harmon MB, Murray T, et al. Cancer statistics, 2001. CA Cancer J Clin. 2001;51:15-36.

6. Fagundes H, Perez CA, Grigsby PW, et aI. Distant metastases after irradiation alone in carcinoma of the uterine cervix. International Journal of radiation oncology, biology, physics. 1992 Jan;24(2):197-204.

7. Grigsby PW, Perez CA, Chao KSC, et aI. Radiation therapy for carcinoma of the cervix with biopsy-proven positive para-aortic lymph nodes. International Journal of Radiation Oncology* Biology* Physics. 2001 Mar;49(3):733-8

8. Grigsby PW, Vest ML, Perez CA. Recurrent carcinoma of the cervix exclusively in the para aortic nodes following radiation therapy. International journal of radiation oncology,biology,phys ics. 1993 Jan;28(2):451-5.

9. Perez CA, Grigsby PW, Chao KSC, et aI. Tumor size, irradiation dose, and long-term outcome of carcinoma of uterine cervix. International journal of radiation oncology, biology,physics. 1998 May;41(2):307-17.

10. Somrners GM, Grigsby PW, Perez CA, et al. Outcome of recurrent cervical carcinoma following definitive irradiation. Gynecol Oncol. 1989;35:150 -5.

11. Hong JH, Tsai CS, Chang JT, et aI. The prognostic significance of pre and post- treatment SCC levels in patients with squamous cell carcinoma of the cervix treated by radiotherapy. International journal of radiation oncology, biology,physics. 1997 Jan;39(21001):172.

12. Brady LW, Perez CA, Bedwinek JM. Failure patterns in gynecologic cancer. Int J Radiat Oncol Biol Phys. 1986;12:549-57.

13. Wang CJ, Lai CH, Huang HJ, et aI. Recurrent cervical carcinoma after primary radical surgery. Am J Obstet GynecoI. 1999;181:518 -24 .

14. Lai CH, Chang HC, Chang TC, et aI. Prognostic factors and impacts of adjuvant therapy in early -stage cervical carcinoma with pelvic node metastases. Gynecol Oncol. 1993; 51:390-396.

15. Harnmond JA, Herson J, Freedman RS, et aI. The impact of 447 Isolated para aortic node recurrence after radiotherapy $\mathrm{c}$ H.-H. CHOU et aI. Lymph node status on survival in cervical carcinoma. International journal of radiation oncology,biology, $\mathrm{P}$ hysics. 1981;7:1713-18.

16. Maneo A. Landoni F. Cormio G., et aI. Concurrent carboplatin/ 5-fluorouracil and radiotherapy for recurrent cervical carcinoma. Ann OncoI. 1999;10:803-7.

17. Carl UM, Bahnsen J, Rapp W. Radiation therapy of paraaortic lymph nodes in gynaecologic cancers: Techniques, results, and complications. Strahlenther Onkol. 1992;168:383-9.

18. Montana GS, Martz KL, Hanks GE. Patterns and sites of failure in cervix cancer treated in the U.S.A. in 1978. International journal of radiation oncology, biology,physics. 1991 Jan;20(1):87-93. 
19. Nguyen HN, Nordqvist SR. Chemotherapy of advanced and recurrent cervical carcinoma. Semin Surg Oncol. 1999;16:247-50.

20. Paunier JP, Delclos L, Fletcher GH. Cause, time of death, and sites of failure in squamous-cell carcinoma of the uterine cervix on intact uterus. Radiology. 1967 Mar;88(3):555-62.

21. Rotman M, Choi K, Guze C, Racial MV, Hornback N, John M. Prophylactic irradiation of the para-aortic lymph node chain in stage II B and bulky stage IB carcinoma of the cervix, initial treatment results of RTOG 7920.. 1990;19: 513-21.
22. Rotman M, Choi K, Guse C, et al. Prophylactic irradiation of the para- aortic lymph node chain in stage IIB and bulky stage IB carcinoma of the cervix, initial treatment results of RTOG 7920. International journal of radiation oncology,biology,physics. 1990 Sep;19(3):513-21.

23. Haie C, Pejovic MH, Gerbaulet A, et al. Is prophylactic para-aortic irradiation worthwhile in the treatment of advanced cervical carcinoma? Results of a controlled clinical trial of the EORTC radiotherapy group. Radiother Oncol. 1988;11:101-12. 